Pacific Journal of 


\title{
PRODUCT INTEGRALS AND INVERSES IN NORMED RINGS
}

\author{
JON C. HELTON
}

This paper concerns product integrals of functions with values in a normed complete ring. The inverses of elements obtained as such integrals are investigated. In particular, the conditions under which $\left[{ }_{x} \Pi^{y}(1+G)\right]^{-1}$ exists are shown to be related to the requirement that $\int_{x}^{y}\left|G^{2}\right|=0$. Since the existence of $\left[{ }_{x} \Pi^{y}(1+G)\right]^{-1}$ is connected with the existence of the product integrals ${ }_{y} \Pi^{x}(1+G)$ and ${ }_{x} \Pi^{y}(1-G)$, the study of the inverse leads to a study of the conditions under which these integrals exist when ${ }_{x} \Pi^{y}(1+G)$ is known to exist. Commutative and noncommutative rings are considered.

II. Definitions. All integrals and definitions are of the subdivision-refinement type; functions are from $S \times S$ to $N$, where $S$ denotes a linearly ordered set and $N$ denotes a ring which has a multiplicative identity element represented by 1 and has a norm $|\cdot|$ with respect to which $N$ is complete and $|1|=1$. The statements that $G$ is bounded, $G \in O P^{\circ}, G \in O P^{\prime}$, and $G \in O B^{\circ}$ on $\{a, b\}$ mean there exist a subdivision $D$ of $\{a, b\}$ and a number $B$ such that if $J=\left\{x_{q}\right\}_{q=0}^{n}$ is a refinement of $D$, then

(1) $|G(u)|<B$ for $u \in J(I)$,

(2) $\left|\prod_{q=i}^{j}\left(1+G_{q}\right)\right|<B$ for $1 \leqq i \leqq j \leqq n$,

(3) $\left|\prod_{J(I)}(1+G)\right|<B$, and

(4) $\sum_{J(I)}|G|<B$, respectively, where $G_{q}=G\left(x_{q-1}, x_{q}\right)$ and $J(I)=\left\{\left(x_{q-1}, x_{q}\right)\right\}_{q=1}^{n}$. Also,

(1) $G \in O A^{\circ}$ on $\{a, b\}$ only if $\int_{a}^{b} G$ exists and $\int_{a}^{b}\left|G-\int G\right|=0$, and

(2) $G \in O M^{\circ}$ on $\{a, b\}$ only if ${ }_{x}^{a} \Pi^{y}(1+G)$ exists for each subdivision $\{a, x, y, b\}$ of $\{a, b\}$ and $\int_{a}^{b}|1+G-\Pi(1+G)|=0$.

The statement that $G \in O D^{\circ}$ on $\{a, b\}$ means if $\varepsilon>0$ then there exists a subdivision $D$ of $\{a, b\}$ such that if $\left\{x_{q}\right\}_{q=0}^{n}$ is a refinement of $D$ and $1 \leqq i \leqq j \leqq n$, then

$$
\left|1-\left[\prod_{q=i}^{j}\left(1+G_{q}\right)\right]\left[\prod_{q=i}^{j}\left(1-G_{j+i-q}\right)\right]\right|<\varepsilon
$$

and

$$
\left|1-\left[\prod_{q=i}^{i}\left(1-G_{j+i-q}\right)\right]\left[\prod_{q=i}^{j}\left(1+G_{q}\right)\right]\right|<\varepsilon .
$$

If $N$ is commutative, then the preceding inequalities are equivalent 
to the requirement that

$$
\left|1-\prod_{q=i}^{j}\left(1-G_{q}^{2}\right)\right|<\varepsilon .
$$

In the following treatment it is assumed that $\{a, b\}$ is in the linear ordering of $S$. Thus, if $\left\{x_{q}\right\}_{q=0}^{n}$ is a subdivision of $\{a, b\}$, then

(1) $\int_{a}^{b} G \sim \sum_{q=1}^{n} G\left(x_{q-1}, x_{q}\right)$,

(2) $\int_{b}^{a} G \sim \sum_{q=1}^{n} G\left(x_{n+1-q}, x_{n-q}\right)$,

(3) ${ }_{a} \prod^{b}(1+G) \sim \prod_{q=1}^{n}\left[1+G\left(x_{q-1}, x_{q}\right)\right]$, and

(4) ${ }_{b} \Pi^{a}(1+G) \sim \prod_{q=1}^{n}\left[1+G\left(x_{n+1-q}, x_{n-q}\right)\right]$.

Similar considerations hold for $O P^{\circ}, O P^{\prime}$, and $O B^{\circ}$. Note that if $G(x, y)=-G(y, x)$ for each subdivision $\{a, x, y, b\}$ of $\{a, b\}$, then

$$
\prod_{q=1}^{n}\left[1+G\left(x_{n+1-q}, x_{n-q}\right)\right]=\prod_{q=1}^{n}\left[1-G\left(x_{n-q}, x_{n+1-q}\right)\right] .
$$

We adopt the conventions that $G(x, x)=0$ and $\Pi_{q=r}^{s}\left(1+G_{q}\right)=1$ if $r>s$. See B. W. Helton [2] and J. S. MacNerney [5] for additional details.

III. Results: noncommutative rings.

Lemma 3.1. If $\left\{a_{q}\right\}_{q=1}^{n}$ is a sequence of elements of $N$, then

$$
\begin{aligned}
\mid 1- & {\left[\prod_{q=1}^{n}\left(1+a_{q}\right)\right]\left[\prod_{q=1}^{n}\left(1-a_{n+1-q}\right)\right] \mid } \\
& \leqq \sum_{q=1}^{n}\left[\left|a_{q}^{2}\right|\right]\left[\left|\prod_{j=1}^{q}\left(1+a_{j}\right)\right|\right]\left[\left|\prod_{j=2}^{q}\left(1-a_{q+1-j}\right)\right|\right] .
\end{aligned}
$$

Indication of proof. Lemma 3.1 can be established by induction.

LEMMA 3.2. If $G$ is a function from $S \times S$ to $N$ such that $\int_{a}^{b}\left|G^{2}\right|=0, G \in O P^{\circ}$ on $\{a, b\}$ and $\{b, a\}$ and $G(x, y)=-G(y, x)$ for each subdivision $\{a, x, y, b\}$ of $\{a, b\}$, then $G \in O D^{\circ}$ on $\{a, b\}$.

Indication of proof. Lemma 3.2 follows as a corollary to Lemma 3.1 .

Lemma 3.3. If $G$ is a function from $S \times S$ to $N$ such that $\int_{a}^{b}\left|G^{2}\right|=$ $0,{ }_{a} \Pi^{b}(1+G)$ exists, $G \in O P^{\circ}$ on $\{a, b\}$ and $\{b, a\}, G(x, y)=-G(y, x)$ for each subdivision $\{a, x, y, b\}$ of $\{a, b\}$ and $\varepsilon>0$, then there exists a subdivision $D$ of $\{a, b\}$ such that if $\left\{x_{q}\right\}_{q=0}^{n}$ and $\left\{y_{q}\right\}_{q=0}^{m}$ are refinements of $D$, then 


$$
\left|1-\left\{\prod_{q=1}^{n}\left[1+G\left(x_{q-1}, x_{q}\right)\right]\right\}\left\{\prod_{q=1}^{m}\left[1-G\left(y_{m-q}, y_{m+1-q}\right)\right]\right\}\right|<\varepsilon
$$

and

$$
\left|1-\left\{\prod_{q=1}^{m}\left[1-G\left(y_{m-q}, y_{m+1-q}\right)\right]\right\}\left\{\prod_{q=1}^{n}\left[1+G\left(x_{q-1}, x_{q}\right)\right]\right\}\right|<\varepsilon .
$$

Indication of proof. Lemma 3.3 follows by using Lemma 3.2 and the Cauchy criterion for product integrals.

LEMMA 3.4. If $G$ is a function from $S \times S$ to $N$ such that $G \in$ $O D^{\circ}$ on $\{a, b\}, G \in O P^{\prime}$ on $\{a, b\}$ and $\{b, a\},{ }_{a} \Pi^{b}(1+G)$ exists, ${ }_{b} \Pi^{a}(1+G)$ exists and $G(x, y)=-G(y, x)$ for each subdivision $\{a, x, y, b\}$ of $\{a, b\}$, then $\left[\Pi_{a}^{b}(1+G)\right]^{-1}$ exists and is ${ }_{b} \Pi^{a}(1+G)$.

Proof. Let $\varepsilon>0$. There exists a subdivision $D$ of $\{a, b\}$ and a number $B$ such that if $\left\{x_{q}\right\}_{q=0}^{n}$ is a refinement of $D$, then

(1) $\left|\prod_{q=1}^{n}\left(1+G_{q}\right)\right|<B$,

(2) $\left|\prod_{q=1}^{n}\left(1-G_{n+1-q}\right)\right|<B$,

(3) $\left|1-\left[\prod_{q=1}^{n}\left(1+G_{q}\right)\right]\left[\prod_{q=1}^{n}\left(1-G_{n+1-q}\right)\right]\right|<\varepsilon / 4$,

(4) $\left|{ }_{a} \Pi^{b}(1+G)-\Pi_{q=1}^{n}\left(1+G_{q}\right)\right|<\varepsilon(4 B)^{-1}$

(5) $\left|{ }_{b} \Pi^{a}(1+G)-\prod_{q=1}^{n}\left(1-G_{n+1-q}\right)\right|<\varepsilon(4 B)^{-1}$, and

(6) $\left[\varepsilon(4 B)^{-1}\right]^{2}<\varepsilon / 4$.

Suppose $\left\{x_{q}\right\}_{q=0}^{n}$ is a refinement of $D$. Let $P_{1}$ and $P_{2}$ denote $\prod_{q=1}^{n}\left(1+G_{q}\right)$ and $\prod_{q=1}^{n}\left(1-G_{n+1-q}\right)$, respectively. Thus,

$$
\begin{aligned}
\mid 1 & -\left[{ }_{a} \Pi^{b}(1+G)\right]\left[{ }_{b} \Pi^{a}(1+G)\right] \mid \\
& \leqq\left|1-P_{1} P_{2}\right|+\left|{ }_{a} \Pi^{b}(1+G)-P_{1}\right|\left|{ }_{b} \Pi^{a}(1+G)-P_{2}\right| \\
& +\left|{ }_{a} \Pi^{b}(1+G)-P_{1}\right|\left|P_{2}\right|+\left|{ }_{b} \Pi^{a}(1+G)-P_{2}\right|\left|P_{1}\right| \\
& <\varepsilon / 4+\left[\varepsilon(4 B)^{-1}\right]^{2}+B\left[\varepsilon(4 B)^{-1}\right]+B\left[\varepsilon(4 B)^{-1}\right]<\varepsilon .
\end{aligned}
$$

Therefore, $\left[{ }_{a} \Pi^{b}(1+G)\right]^{-1}$ exists and is ${ }_{b} \Pi^{a}(1+G)$.

THEOREM 3.1. If $G$ is a function from $S \times S$ to $N$ such that $\int_{a}^{b}\left|G^{2}\right|=0,{ }_{a} \Pi^{b}(1+G)$ exists, $G \in O P^{\circ}$ on $\{a, b\}$ and $\{b, a\}$ and $G(x, y)=$ - $G(y, x)$ for each subdivision $\{a, x, y, b\}$ of $\{a, b\}$, then

(1) ${ }_{b} \Pi^{a}(1+G)$ exists, and

(2) $\left[{ }_{a} \Pi^{b}(1+G)\right]^{-1}$ exists and is ${ }_{b} \Pi^{a}(1+G)$.

Proof. We initially use the Cauchy criterion to show that ${ }_{b} \Pi^{a}(1+G)$ exists. Let $\varepsilon>0$. There exists a subdivision $D_{1}$ of $\{a, b\}$ and a number $B$ such that if $\left\{x_{q}\right\}_{q=0}^{n}$ is a refinement of $D_{1}$, then

$$
\left|\prod_{q=1}^{n}\left(1+G_{q}\right)\right|<B
$$


and

$$
|-1|\left|\prod_{q=1}^{n}\left(1-G_{n+1-q}\right)\right|<B .
$$

Lemma 3.3 implies that there exists a subdivision $D_{2}$ of $\{a, b\}$ such that if $\left\{x_{q}\right\}_{q=0}^{n}$ and $\left\{y_{q}\right\}_{q=0}^{m}$ are refinements of $D_{2}$, then

$$
\left|1-\left\{\prod_{q=1}^{n}\left[1+G\left(x_{q-1}, x_{q}\right)\right]\right\}\left\{\prod_{q=1}^{m}\left[1-G\left(y_{m-q}, y_{m+1-q}\right)\right]\right\}\right|<\varepsilon(2 B)^{-1}
$$

and

$$
\left|1-\left\{\prod_{q=1}^{m}\left[1-G\left(y_{m-q}, y_{m+1-q}\right)\right]\right\}\left\{\prod_{q=1}^{n}\left[1+G\left(x_{q-1}, x_{q}\right)\right]\right\}\right|<\varepsilon(2 B)^{-1} .
$$

Let $D=D_{1} \cup D_{2}$ and suppose $\left\{x_{q}\right\}_{q=0}^{n}$ and $\left\{y_{q}\right\}_{q=0}^{m}$ are refinements of $D$. Let $P_{1}, P_{2}$, and $P_{3}$ denote

$$
\begin{gathered}
\prod_{q=1}^{n}\left[1+G\left(x_{q-1}, x_{q}\right)\right], \\
\prod_{q=1}^{n}\left[1-G\left(x_{n-q}, x_{n+1-q}\right)\right],
\end{gathered}
$$

and

$$
\prod_{q=1}^{m}\left[1-G\left(y_{m-q}, y_{m+1-q}\right)\right]
$$

respectively. Now,

$$
\begin{aligned}
\varepsilon / 2 & >\left[\left|P_{3}\right|\right]\left[\varepsilon(2 B)^{-1}\right] \\
& \geqq\left|P_{3}\right|\left|1-P_{1} P_{2}\right| \\
& \geqq\left|P_{3}-P_{3} P_{1} P_{2}\right| \\
& \geqq\left|P_{3}-P_{2}\right|-\left|1-P_{3} P_{1}\right|\left|P_{2}\right||-1| \\
& >\left|P_{3}-P_{2}\right|-B\left[\varepsilon(2 B)^{-1}\right] .
\end{aligned}
$$

Therefore, $\varepsilon>\left|P_{3}-P_{2}\right|$, and hence, ${ }_{b} \Pi^{a}(1+G)$ exists.

Lemma 3.2 implies that $G \in O D^{\circ}$ on $\{a, b\}$. Hence, it follows from Lemma 3.4 that $\left[{ }_{a} \Pi^{b}(1+G)\right]^{-1}$ exists and is ${ }_{b} \Pi^{a}(1+G)$.

Lemma 3.5. If $G$ is a bounded function from $S \times S$ to $N$ such that $\int_{a}^{b}\left|G^{2}\right|=0, G \in O M^{\circ}$ on $\{a, b\},{ }_{y} \Pi^{x}(1+G)$ exists and is

$$
\left[{ }_{x} \Pi^{y}(1+G)\right]^{-1}
$$

for each subdivision $\{a, x, y, b\}$ of $\{a, b\}, G(x, y)=-G(y, x)$ for each subdivision $\{a, x, y, b\}$ of $\{a, b\}$, and $\left[{ }_{x} \Pi^{y}(1+G)\right]^{-1}$ is bounded on $\{a, b\}$, then $G \in O M^{\circ}$ on $\{b, a\}$. 
Proof. Since we are given that ${ }_{y} \Pi^{x}(1+G)$ exists for each subdivision $\{a, x, y, b\}$ of $\{a, b\}$, it is only necessary to show that

$$
\int_{b}^{a}|1+G-\Pi(1+G)|=0 .
$$

Let $\varepsilon>0$. There exist a subdivision $D$ of $\{a, b\}$ and a number $B$ such that if $\left\{x_{q}\right\}_{q=0}^{n}$ is a refinement of $D$, then

(1) $\left|1-G_{q}\right|<B$ for $1 \leqq q \leqq n$,

(2) $\left|\left[_{x_{q-1}} \Pi^{x_{q}}(1+G)\right]^{-1}\right|<B$ for $1 \leqq q \leqq n$,

(3) $\sum_{q=1}^{n}\left|\left[x_{q-1} \Pi^{x_{q}}(1+G)\right]-\left[1+G_{q}\right]\right|<\varepsilon\left(2 B^{2}\right)^{-1}$, and

(4) $\quad \sum_{q=1}^{n}\left|-G_{q}^{2}\right|<\varepsilon(2 B)^{-1}$.

Let $\left\{x_{q}\right\}_{q=0}^{n}$ be a refinement of $D$ and suppose $P_{q}$ denotes $x_{q-1} \Pi^{x_{q}}(1+G)$ for $1 \leqq q \leqq n$. Thus,

$$
\begin{aligned}
\sum_{q=1}^{n} \mid[ & \left.-G_{q}\right]-\left[x_{q} \Pi^{x_{q-1}}(1+G)\right] \mid \\
& =\sum_{q=1}^{n}\left|\left\{\left[1-G_{q}\right]-\left[P_{q}\right]^{-1}\right\}\left\{\left[P_{q}\right]\left[P_{q}\right]^{-1}\right\}\right| \\
& \leqq \sum_{q=1}^{n}\left|\left[1-G_{q}\right]\left[P_{q}\right]-1\right|\left|\left[P_{q}\right]^{-1}\right| \\
& \leqq B \sum_{q=1}^{n}\left|\left[1-G_{q}\right]\left[P_{q}-\left(1+G_{q}\right)+\left(1+G_{q}\right)\right]-1\right| \\
& \leqq B^{2} \sum_{q=1}^{n}\left|P_{q}-\left(1+G_{q}\right)\right|+B \sum_{q=1}^{n}\left|-G_{q}^{2}\right| \\
& <B^{2}\left[\varepsilon\left(2 B^{2}\right)^{-1}\right]+B\left[\varepsilon(2 B)^{-1}\right]=\varepsilon .
\end{aligned}
$$

Therefore, $G \in O M^{\circ}$ on $\{b, a\}$.

The proof of Lemma 3.5 is essentially the same as the proof of a previous result by the author [3, Theorem 4]. However, since the argument is relatively brief and the setting here is somewhat different from that in [3], the proof is included for completeness.

THEOREM 3.2. If $G$ is a function from $S \times S$ to $N$ such that $\int_{a}^{b}\left|G^{2}\right|=0, G \in O P^{\circ}$ on $\{a, b\}$ and $\{b, a\}, G \in O M^{\circ}$ on $\{a, b\}$ and $G(x, y)=$ $-G(y, x)$ for each subdivision $\{a, x, y, b\}$ of $\{a, b\}$, then $G \in O M^{\circ}$ on $\{b, a\}$.

Proof. It follows from Theorem 3.1 that ${ }_{y} \Pi^{x}(1+G)$ exists and is $\left[{ }_{x} \Pi^{y}(1+G)\right]^{-1}$ for each subdivision $\{a, x, y, b\}$ of $\{a, b\}$. Therefore, Theorem 3.2 follows from Lemma 3.5.

We now show that under certain restrictions the Riccati integral equation

$$
n(y)=k+\int_{a}^{y} n(r) G(r, s) n(r)
$$


has a solution in a noncommutative ring. Initially, we state two lemmas on the product integral solution of integral equations. Both of these lemmas are based on a result of B. W. Helton [2, Theorem 5.1, p. 310]. The hypothesis of this result has been modified to produce the lemmas given here. However, with the use of Theorem 3.1 to assure the existence of certain inverses, these lemmas can be established by arguments that are essentially the same as the one given by $\mathrm{B} . \mathrm{W}$. Helton.

LEMMA 3.6. If

(a) $a \in S, k \in N, k^{-1}$ exists and $f$ is a function from $S$ to $N$ such that $f(a)=k$, and

(b) $G$ is a function from $S \times S$ to $N$ such that if $\{a, y\} \in S \times S$, then $G \in O P^{\circ}$ on $\{a, y\}$ and $\{y, a\}, G(u, v)=-G(v, u)$ for each subdivi$\operatorname{sion}\{a, u, v, y\}$ of $\{a, y\}$ and $\int_{a}^{y}\left|G^{2}\right|=0$,

then the following statements are equivalent:

(1) if $\{a, y\} \in S \times S$, then $f(r) G(r, s) \in O A^{\circ}$ on $\{a, y\}$ and

$$
f(y)=k+\int_{a}^{y} f(r) G(r, s)
$$

and

(2) if $\{a, y\} \in S \times S$, then $G \in O M^{\circ}$ on $\{a, y\}$ and

$$
f(y)=k\left[{ }_{a} \Pi^{y}(1+G)\right] \text {. }
$$

LEMMA 3.7. If

(a) $a \in S, k \in N, k^{-1}$ exists and $f$ is a function from $S$ to $N$ such that $f(a)=k$, and

(b) $G$ is a function from $S \times S$ to $N$ such that if $\{y, a\} \in$ $S \times S$, then $G \in O P^{\circ}$ on $\{y, a\}$ and $\{a, y\}, G(u, v)=-G(v, u)$ for each subdivision $\{y, u, v, a\}$ of $\{y, a\}$ and $\int_{y}^{a}\left|G^{2}\right|=0$, then the following statements are equivalent:

(1) if $\{y, a\} \in S \times S$, then $G(r, s) f(s) \in O A^{\circ}$ on $\{y, a\}$ and

$$
f(y)=k+\int_{y}^{a} G(r, s) f(s),
$$

and

(2) if $\{y, a\} \in S \times S$, then $G \in O M^{\circ}$ on $\{y, a\}$ and

$$
f(y)=\left[{ }_{y} \Pi^{a}(1+G)\right] k .
$$

We now establish the integral equation result. The proof presented here was suggested by B. W. Helton's proof for the commutative case [2, Examples 4,5, p. 320]. 
THEOREM 3.3. If

(a) $a \in S, k \in N, k^{-1}$ exists and $n$ is a function from $S$ to $N$ such that $n(a)=k$,

(b) $G$ is a function from $S \times S$ to $N$ such that if $\{a, y\} \in S \times S$, then $G(r, s) n(r) \in O P^{\circ}$ on $\{a, y\}, G(r, s) n(s) \in O P^{\circ}$ on $\{y, a\}, G(u, v)=-G(v, u)$ for each subdivision $\{a, u, v, y\}$ of $\{a, y\}$ and

and

$$
\int_{a}^{y}\left|[G(r, s) n(r)]^{2}\right|=0
$$

(c) if $\{a, y\} \in S \times S$, then $n(r) G(r, s) n(r) \in O A^{\circ}$ on $\{a, y\}$ and

$$
n(y)=k+\int_{a}^{y} n(r) G(r, s) n(r),
$$

then (conclusion) if $\{a, y\} \in S \times S$,

$$
n(y)=\left[k^{-1}+\int_{y}^{a} G\right]^{-1} .
$$

Proof. If $\{a, y\} \in S \times S$ and $H$ denotes the function such that if $\{a, r, s, y\}$ is a subdivision of $\{a, y\}$, then

(1) $H(r, s)=G(r, s) n(r)$, and

(2) $H(s, r)=G(s, r) n(r)$,

then we know the following about $H: H \in O P^{\circ}$ on $\{a, y\}$ and $\{y, a\}$, $H(u, v)=-H(v, u)$ for each subdivision $\{a, u, v, y\}$ of $\{a, y\}, \int_{a}^{y}\left|H^{2}\right|=$ $0, n(r) H(r, s) \in O A^{\circ}$ on $\{a, y\}$ and

$$
n(y)=k+\int_{a}^{y} n(r) H(r, s) .
$$

Therefore, it follows from Lemma 3.6 that if $\{a, y\} \in S \times S$, then $H \in$ $O M^{\circ}$ on $\{a, y\}$ and

$$
n(y)=k\left[\Pi_{a} \Pi^{y}(1+H)\right] .
$$

Again, if we suppose $\{a, y\} \in S \times S$ and define $H$ as before, then we know the following about $H: H \in O P^{\circ}$ on $\{a, y\}$ and $\{y, a\}, H(u, v)=$ - $H(v, u)$ for each subdivision $\{a, u, v, y\}$ of $\{a, y\}, \int_{a}^{y}\left|H^{2}\right|=0$ and $H \in O M^{\circ}$ on $\{a, y\}$. Thus, from Theorem 3.1, $\left[{ }_{a} \Pi^{y}(1+H)\right]^{-1}$ exists and is ${ }_{y} \Pi^{a}(1+H)$. Further, from Theorem 3.2, $H \in O M^{\circ}$ on $\{y, a\}$. Now, since $k^{-1}$ exists,

$$
\begin{aligned}
{[n(y)]^{-1} } & =\left\{k\left[\left[_{a} \Pi^{y}(1+H)\right]\right\}^{-1}\right. \\
& =\left[{ }_{y} \Pi^{a}(1+H)\right] k^{-1} .
\end{aligned}
$$

Once more, if we suppose $\{a, y\} \in S \times S$ and define $H$ as before, then we know the following about $H: H \in O P^{\circ}$ on $\{a, y\}$ and $\{y, a\}$, 
$H(u, v)=-H(v, u)$ for each subdivision $\{y, u, v, a\}$ of $\{y, a\}, \int_{y}^{a}\left|G^{2}\right|=$ $0, H \in O M^{\circ}$ on $\{y, a\}$ and

$$
[n(y)]^{-1}=\left[{ }_{y} \Pi^{a}(1+H)\right] k^{-1} .
$$

Thus, from Lemma 3.7,

$$
\begin{aligned}
{[n(y)]^{-1} } & =k^{-1}+\int_{y}^{a}[H(r, s)][n(s)]^{-1} \\
& =k^{-1}+\int_{y}^{a}[G(r, s) n(s)][n(s)]^{-1} \\
& =k^{-1}+\int_{y}^{a} G .
\end{aligned}
$$

We have now established that if $\{a, y\} \in S \times S$, then

$$
n(y)=\left[k^{-1}+\int_{y}^{a} G\right]^{-1} .
$$

IV. Results: commutative rings.

Theorem 4.1. Suppose $N$ is commutative. If $G$ is a function from $S \times S$ to $N$ such that $G \in O D^{\circ}$ on $\{a, b\},{ }_{a} \Pi^{b}(1+G)$ exists and $-G \in O P^{\prime}$ on $\{a, b\}$, then

(1) ${ }_{a} \Pi^{b}(1-G)$ exists, and

(2) $\left[\Pi_{a}^{b}(1+G)\right]^{-1}$ exists and is ${ }_{a} \Pi^{b}(1-G)$.

Proof. We use the Cauchy criterion for product integrals to show that ${ }_{a} \Pi^{b}(1-G)$ exists. Let $\varepsilon>0$. There exist a subdivision $D$ of $\{a, b\}$ and a number $B$ such that if $K$ and $L$ are refinements of $D$, then

(1) $\left|\Pi_{K(I)}(1+G)\right|<B$ and $\left|\Pi_{K(I)}(1-G)\right|<B$,

(2) $\left|\prod_{K(I)}(1+G)-\Pi_{L(I)}(1+G)\right|<\varepsilon\left(3 B^{2}\right)^{-1}$, and

(3) $\left|1-\Pi_{K(I)}\left(1-G^{2}\right)\right|<\varepsilon(6 B)^{-1}$.

Suppose $K$ and $L$ are refinements of $D$. Let $P_{1}, P_{2}, P_{3}$, and $P_{4}$ denote $\Pi_{K(I)}(1-G), \Pi_{L(I)}(1-G), \Pi_{K^{(I)}}\left(1-G^{2}\right)$, and $\prod_{L(I)}\left(1-G^{2}\right)$, respectively. Thus,

$$
\begin{aligned}
\left|P_{1}-P_{2}\right|= & \left|\left\{P_{1}-P_{2}\right\}\left\{P_{3}+\left[1-P_{3}\right]\right\}\right| \\
< & \left|P_{1} P_{3}-P_{2} P_{3}\right|+[2 B]\left[\varepsilon(6 B)^{-1}\right] \\
\leqq & \left|P_{1} P_{3}-P_{1} P_{4}\right|+\left|P_{1} P_{4}-P_{2} P_{3}\right|+\varepsilon / 3 \\
\leqq & \left|P_{1}\right|\left[\left|P_{3}-1\right|+\left|1-P_{4}\right|\right] \\
& +\left|P_{1}\right|\left|P_{2}\right|\left|\Pi_{L(I)}(1+G)-\prod_{K(I)}(1+G)\right|+\varepsilon / 3 \\
< & B\left[\varepsilon(6 B)^{-1}\right]+\varepsilon(6 B)^{-1}+B^{2}\left[\varepsilon\left(3 B^{2}\right)^{-1}\right]+\varepsilon / 3 \\
= & \varepsilon .
\end{aligned}
$$


Therefore, ${ }_{a} \Pi^{b}(1-G)$ exists.

Since $G \in O D^{\circ}$ on $\{a, b\}$, it follows readily that $\left[{ }_{a} \Pi^{b}(1+G)\right]^{-1}$ exists and is ${ }_{a} \Pi^{b}(1-G)$.

LEMMA 4.1. Suppose $N$ is commutative. If $G$ is a function from $S \times S$ to $N$, then the following statements are equivalent:

(1) $G \in O D^{\circ}$ on $\{a, b\}$ and $-G^{2} \in O M^{\circ}$ on $\{a, b\}$, and

(2) $\int_{a}^{b}\left|G^{2}\right|=0$.

Proof $(1 \rightarrow 2)$. Since $G \in O D^{\circ}$ on $\{a, b\},{ }_{x} \Pi^{y}\left(1-G^{2}\right)$ exists and is 1 for each subdivision $\{a, x, y, b\}$ of $\{a, b\}$. Thus, since $-G^{2} \in O M^{\circ}$ on $\{a, b\}$,

$$
\begin{aligned}
0 & =\int_{a}^{b}\left|\Pi\left(1-G^{2}\right)-\left(1-G^{2}\right)\right| \\
& =\int_{a}^{b}\left|1-\left(1-G^{2}\right)\right|=\int_{a}^{b}\left|G^{2}\right| .
\end{aligned}
$$

Proof $(2 \rightarrow 1)$. Note that $\left|-G^{2}\right| \in O P^{\circ}$ on $\{a, b\}$. There exist a subdivision $D$ of $\{a, b\}$ and a number $B$ such that if $\left\{x_{q}\right\}_{q=0}^{n}$ is a refinement of $D$, then

(1) $\Pi_{q=i}^{j}\left(1+\left|-G_{q}^{2}\right|\right)<B$ for $1 \leqq i \leqq j \leqq n$, and

(2) $\sum_{q=1}^{n}\left|G_{q}^{2}\right|<\varepsilon / B$.

Suppose $\left\{x_{q}\right\}_{q=0}^{n}$ is a refinement of $D$. Thus, if $1 \leqq i \leqq j \leqq n$, then

$$
\begin{aligned}
\mid 1- & \prod_{q=i}^{j}\left(1-G_{q}^{2}\right) \mid \\
& =\left|1-\left\{1+\sum_{q=i}^{j}\left[-G_{q}^{2}\right]\left[\prod_{k=q+1}^{j}\left(1-G_{k}^{2}\right)\right]\right\}\right| \\
& \leqq \sum_{q=i}^{j}\left[\left|G_{q}^{2}\right|\right]\left[\prod_{k=q+1}^{j}\left(1+\left|-G_{k}^{2}\right|\right)\right] \\
& <B \varepsilon / B=\varepsilon .
\end{aligned}
$$

Therefore, $G \in O D^{\circ}$ on $\{a, b\}$.

Since $\int_{a}^{b}\left|G^{2}\right|=0$, it follows that $-G^{2} \in O A^{\circ}$ and $O B^{\circ}$ on $\{a, b\}$. Therefore, ${ }^{2}-G^{2} \in O M^{\circ}$ on $\{a, b\}$ by a result of B. W. Helton [2, Theorem 3.4, p. 301].

LeMma 4.2. Suppose $N$ is commutative. If $G$ is a function from $S \times S$ to $N$ such that $G \in O D^{\circ}$ and $O P^{\circ}$ on $\{a, b\}$ and $-G \in O P^{\prime}$ on $\{a, b\}$, then $-G \in O P^{\circ}$ on $\{a, b\}$.

Proof. There exist a subdivision $H=\left\{y_{q}\right\}_{q=0}^{m}$ of $\{a, b\}$ and a number $B>1$ such that if $J=\left\{x_{q}\right\}_{q=0}^{n}$ is a refinement of $H$, then 
(1) $\left|\Pi_{J(I)}(1-G)\right|<B$,

(2) $\left|\prod_{q=i}^{j}\left(1+G_{q}\right)\right|<B$ for $1 \leqq i \leqq j \leqq n$, and

( 3 ) $\left|\prod_{q=i}^{j}\left(1-G_{q}^{2}\right)\right|<B$ for $1 \leqq i \leqq j \leqq n$.

Suppose $-G \notin O P^{\circ}$ on $\{a, b\}$. Hence, there exist sequences $\left\{p_{i}\right\}_{i=1}^{\infty},\left\{q_{i}\right\}_{i=1}^{\infty}$ and $\left\{H_{i}\right\}_{i=1}^{\infty}$ and positive integers $r$ and $s$ such that

(1) $1 \leqq r \leqq s \leqq m,\left\{y_{r-1}, p_{i}, y_{r}\right\}$ is a subdivision of $\left\{y_{r-1}, y_{r}\right\}$ and $\left\{y_{s-1}, q_{i}, y_{s}\right\}$ is a subdivision of $\left\{y_{s-1}, y_{s}\right\}$, and

(2) $H_{i}$ is a subdivision of $\left\{p_{i}, q_{i}\right\}$ such that $i<\left|\prod_{H_{i(I)}}(1-G)\right|$ and if $r<s$ then $\left\{y_{q}\right\}_{q=r}^{s-1} \subseteq H_{i}$.

Let $i$ be a positive integer such that $i>3+B^{3}$. Further, let $P=\left|\Pi_{H_{\boldsymbol{i}}(I)}(1-G)\right|$ and let $J=H \cup H_{i}$. Since $G \in O D^{\circ}$ on $\{a, b\}$, there exist subdivisions $K$ and $L$ of $\left\{a, p_{i}\right\}$ and $\left\{q_{i}, b\right\}$, respectively, such that

(1) $\left\{y_{q}\right\}_{q=0}^{r-1} \subseteq K$ and $\left\{y_{q}\right\}_{q=s}^{m} \subseteq L$,

(2) $|U|<(P B)^{-1}$, where $U=1-\Pi_{K(I)}\left(1-G^{2}\right)$, and

(3) $|V|<(P B)^{-1}$, where $V=1-\Pi_{L(I)}\left(1-G^{2}\right)$. Thus,

$$
\begin{aligned}
& i<\left|\Pi_{H_{i(I)}}(1-G)\right| \\
& =\left|\left[U+\Pi_{K(I)}\left(1-G^{2}\right)\right]\left[\Pi_{H_{i}(I)}(1-G)\right]\left[\Pi_{L(I)}\left(1-G^{2}\right)+V\right]\right| \\
& \leqq|U|\left|\Pi_{H_{i}(I)}(1-G)\right|\left|\Pi_{L(I)}\left(1-G^{2}\right)\right| \\
& +|U|\left|\Pi_{H_{i^{(I)}}}(1-G)\right||V| \\
& +\left|\Pi_{K(I)}(1+G)\right|\left|\Pi_{J(I)}(1-G)\right|\left|\Pi_{L(I)}(1+G)\right| \\
& +\left|\Pi_{K(I)}\left(1-G^{2}\right)\right|\left|\Pi_{H_{\boldsymbol{i}^{(I)}}}(1-G)\right||V| \\
& <(P B)(P B)^{-1}+P(P B)^{-2}+B^{3}+(P B)(P B)^{-1} \\
& <3+B^{3}<i \text {. }
\end{aligned}
$$

This is a contradiction, and therefore, $-G \in O P^{\circ}$ on $\{a, b\}$.

Theorem 4.2. Suppose $N$ is commutative. If $G$ is a function from $S \times S$ to $N$ such that $G \in O P^{\circ}$ and $O M^{\circ}$ on $\{a, b\},-G \in O P^{\prime}$ on $\{a, b\}$ and $\int_{a}^{b}\left|G^{2}\right|=0$, then $-G \in O M^{\circ}$ on $\{a, b\}$.

Proof. It follows from Theorem 4.1 that ${ }_{x} \Pi^{y}(1-G)$ exists and is $\left[{ }_{x} \Pi^{y}(1+G)\right]^{-1}$ for each subdivision $\{a, x, y, b\}$ of $\{a, b\}$. Further, since $G \in O D^{\circ}$ on $\{a, b\}$ by Lemma 4.1 , Lemma 4.2 implies that $-G \in$ $O P^{\circ}$ on $\{a, b\}$. Therefore, it follows from Lemma 3.5 that $-G \in O M^{\circ}$ on $\{a, b\}$.

THEOREM 4.3. Suppose $N$ is commutative. If $G$ is a function from $S \times S$ to $N$ such that ${ }_{x} \Pi^{y}(1+G)$ and ${ }_{x} \Pi^{y}(1-G)$ exist and 
$\left[{ }_{x} \Pi^{y}(1+G)\right]^{-1}$ exists and is ${ }_{x} \Pi^{y}(1-G)$ for each subdivision $\{a, x, y, b\}$ of $\{a, b\}$, then $G \in O D^{\circ}$ on $\{a, b\}$.

Proof. Suppose $G \notin O D^{\circ}$ on $\{a, b\}$. Hence, there exists a positive number $\varepsilon$ such that if $H$ is a subdivision of $\{a, b\}$ then there exist a refinement $\left\{x_{q}\right\}_{q=0}^{n}$ of $H$ and integers $i$ and $j$ such that $1 \leqq i \leqq j \leqq n$ and

$$
\left|1-\prod_{q=i}^{j}\left(1-G_{q}^{2}\right)\right| \geqq \varepsilon .
$$

For convenience, suppose $1>\varepsilon$. Note that ${ }_{x} \Pi^{y}\left(1-G^{2}\right)$ exists and is 1 for each subdivision $\{a, x, y, b\}$ of $\{a, b\}$. Thus, there exists a subdivision $H$ of $\{a, b\}$ such that if $J$ is a refinement of $H$, then

$$
\varepsilon / 4>\left|1-\Pi_{J(I)}\left(1-G^{2}\right)\right| \text {. }
$$

Hence, there exist a refinement $\left\{x_{q}\right\}_{q=0}^{n}$ of $H$ and integers $i$ and $j$ such that $1 \leqq i \leqq j \leqq n$ and

$$
\left|1-\prod_{q=i}^{j}\left(1-G_{q}^{2}\right)\right| \geqq \varepsilon .
$$

Let $K_{1}$ and $K_{2}$ represent $\left\{x_{q}\right\}_{q=0}^{i-1}$ and $\left\{x_{q}\right\}_{q=j}^{n}$, respectively. Further, let

$$
P=\left|\prod_{q=i}^{j}\left(1-G_{q}^{2}\right)\right|+1 \text {. }
$$

There exist refinements $L_{1}$ and $L_{2}$ of $K_{1}$ and $K_{2}$, respectively, such that

(1) $\left|-1+\Pi_{L_{1}(I)}\left(1-G^{2}\right)\right|<\varepsilon(4 P)^{-1}$, and

(2) $\left|-1+\Pi_{L_{2}(I)}\left(1-G^{2}\right)\right|<\varepsilon(4 P)^{-1}$.

Let $J=L_{1} \cup H \cup L_{2}$. Thus,

$$
\begin{aligned}
\varepsilon / 4> & \left|1-\prod_{J(I)}\left(1-G^{2}\right)\right| \\
= & \mid 1-\left[1-1+\Pi_{L_{1}(I)}\left(1-G^{2}\right)\right]\left[\prod_{q=i}^{j}\left(1-G_{q}^{2}\right)\right] \\
& \quad \times\left[1-1+\Pi_{L_{2}(I)}\left(1-G^{2}\right)\right] \mid \\
\geqq & \left|1-\prod_{q=i}^{j}\left(1-G_{q}^{2}\right)\right| \\
& -P\left|-1+\Pi_{L_{2}(I)}\left(1-G^{2}\right)\right|-P\left|1-\Pi_{L_{1}(I)}\left(1-G^{2}\right)\right| \\
& -P\left|-1+\prod_{L_{1}(I)}\left(1-G^{2}\right)\right|\left|1-\prod_{L_{2}(I)}\left(1-G^{2}\right)\right| \\
> & \varepsilon-P\left[\varepsilon(4 P)^{-1}\right]-P\left[\varepsilon(4 P)^{-1}\right]-P\left[\varepsilon(4 P)^{-1}\right]^{2}>\varepsilon / 4 .
\end{aligned}
$$

This is a contradiction, and therefore, $G \in O D^{\circ}$ on $\{a, b\}$.

REMARK 1. B. W. Helton $[2, \S 6]$ gives product integral techniques for solving certain types of integral equations in commutative rings. 
An important condition in this development is that

$$
\left[{ }_{x} \Pi^{y}(1+G)\right]^{-1}={ }_{x} \Pi^{y}(1-G) .
$$

In particular, see Examples 4 and 5 [2, p. 320]. Thus, the results in this section can be applied to the solution of certain Riccati type integral equations.

REMARK 2. Related results are obtained by J. S. MacNerney [5, $\S 7]$. However, in that development the functions under consideration are required to have bounded variation. We do not require bounded variation in this development. However, the functions here are often required to belong to the set $O P^{\circ}$. As noted by B. W. Helton [2, p. 299], the set of functions of bounded variation is a proper subset of the set $O P^{\circ}$. Also, the requirement that $\int_{a}^{b}\left|G^{2}\right|=0$ does not imply that $G$ has bounded variation. W. P. Davis and J. A. Chatfield [1, p. 747] give a function $G$ such that $\int_{a}^{b}\left|G^{2}\right|=0,{ }_{a} \Pi^{b}(1+G)$ exists and is not zero, and $G$ does not have bounded variation. In addition, J. V. Herod [4] has also investigated the existence of inverses in a setting similar to the one studied by MacNerney.

REMARK 3. Related results are also obtained in a previous paper by the author [3, Theorems 2,3,4,5]. However, conditions relating to commutativity or the existence of inverses are required there that are not required here.

\section{REFERENCES}

1. W. P. Davis and J. A. Chatfield, Concerning product integrals and exponentials, Proc. Amer. Math. Soc., 25 (1970), 743-747.

2. B. W. Heiton, Integral equations and product integrals, Pacific J. Math., 16 (1966), 297-322.

3. J. C. Helton, Product integrals, bounds and inverses, Texas J. Sci., 25 (1973), to appear.

4. J. V. Herod, Multiplicative inverses of solutions for Volterra-Stieltjes integral equations, Proc. Amer. Math. Soc., 22 (1969), 650-656.

5. J. S. MacNerney, Integral equations and semigroups, Illinois J. Math., 7 (1963), 148-173.

Received October 31, 1972.

Arizona State Universiti 


\section{PACIFIC JOURNAL OF MATHEMATICS}

\section{EDITORS}

RICHARD ARENS (Managing Editor)

University of California

Los Angeles, California 90024

R. A. BeAumont

University of Washington

Seattle, Washington 98105
J. DugundJI*

Department of Mathematics

University of Southern California

Los Angeles, California 90007

D. Gilbarg and J. Milgram

Stanford University

Stanford, California 94305

\section{ASSOCIATE EDITORS}

E. F. BECKENBACH

B. H. NEUMANN

F. WOLF

K. YoSHIDA

\section{SUPPORTING INSTITUTIONS}

UNIVERSITY OF BRITISH COLUMBIA
CALIFORNIA INSTITUTE OF TECHNOLOGY
UNIVERSITY OF CALIFORNIA
MONTANA STATE UNIVERSITY
UNIVERSITY OF NEVADA
NEW MEXICO STATE UNIVERSITY
OREGON STATE UNIVERSITY
UNIVERSITY OF OREGON
OSAKA UNIVERSITY

UNIVERSITY OF BRITISH COLUMBIA CALIFORNIA INSTITUTE OF TECHNOLOGY UNIVERSITY OF CALIFORNIA MONTANA STATE UNIVERSITY NEW MEXICO STATE UNIVERSITY UNIVERSITY OF OREGON OSAKA UNIVERSITY
UNIVERSITY OF SOUTHERN CALIFORNIA STANFORD UNIVERSITY UNIVERSITY OF TOKYO UNIVERSITY OF UTAH WASHINGTON STATE UNIVERSITY UNIVERSITY OF WASHINGTON AMERICAN MATHEMATICAL SOCIETY NAVAL WEAPONS CENTER

* C. R. DePrima California Institute of Technology, Pasadena, CA 91109, will replace J. Dugundji until August 1974. 


\section{Pacific Journal of Mathematics}

\section{Vol. 51, No. $1 \quad$ November, 1974}

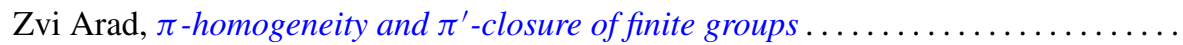

Ivan Baggs, A connected Hausdorff space which is not contained in a maximal

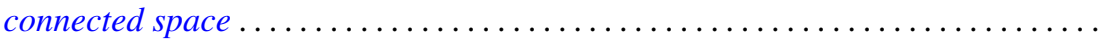

Eric Bedford, The Dirichlet problem for some overdetermined systems on the unit ball in $C^{n}$

R. H. Bing, Woodrow Wilson Bledsoe and R. Daniel Mauldin, Sets generated by

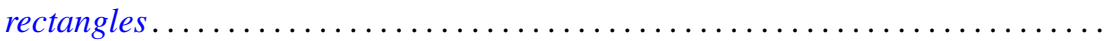

Carlo Cecchini and Alessandro Figà-Talamanca, Projections of uniqueness for

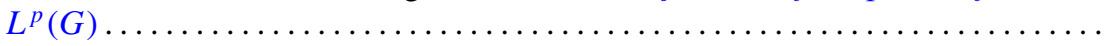

Gokulananda Das and Ram N. Mohapatra, The non absolute Nörlund summability of Fourier series .

Frank Rimi DeMeyer, On separable polynomials over a commutative ring ........ Richard Detmer, Sets which are tame in arcs in $E^{3} \ldots \ldots \ldots \ldots \ldots \ldots \ldots \ldots$

William Erb Dietrich, Ideals in convolution algebras on Abelian groups ..........

Bryce L. Elkins, A Galois theory for linear topological rings .................

William Alan Feldman, A characterization of the topology of compact convergence on $C(X)$.

Hillel Halkin Gershenson, A problem in compact Lie groups and framed cobordism

Samuel R. Gordon, Associators in simple algebras.

Marvin J. Greenberg, Strictly local solutions of Diophantine equations

Jon Craig Helton, Product integrals and inverses in normed rings . . . . . . . . . . . .

Domingo Antonio Herrero, Inner functions under uniform topology . . .

Jerry Alan Johnson, Lipschitz spaces .

Marvin Stanford Keener, Oscillatory solutions and multi-point boundary value

functions for certain nth-order linear ordinary differential equations.

John Cronan Kieffer, A simple proof of the Moy-Perez generalization of the

Shannon-McMillan theorem .......................

Joong Ho Kim, Power invariant rings

Gangaram S. Ladde and V. Lakshmikantham, On flow-invariant sets .

Roger T. Lewis, Oscillation and nonoscillation criteria for some self-adjoint even

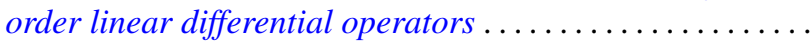

Jürg Thomas Marti, On the existence of support points of solid convex sets ..

John Rowlay Martin, Determining knot types from diagrams of knots . .

James Jerome Metzger, Local ideals in a topological algebra of entire functions

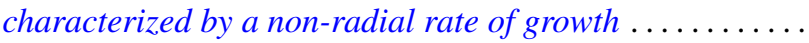

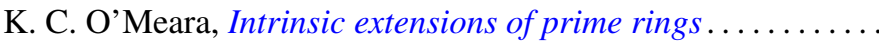

Stanley Poreda, A note on the continuity of best polynomial approximations ..

Robert John Sacker, Asymptotic approach to periodic orbits and local prolongations

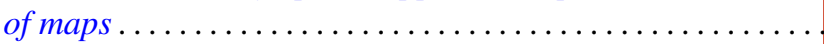

Eric Peter Smith, The Garabedian function of an arbitrary compact set . .

Arne Stray, Pointwise bounded approximation by functions satisfying a side condition

John St. Clair Werth, Jr., Maximal pure subgroups of torsion complete abelian

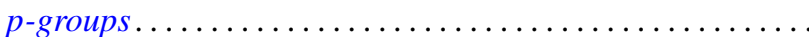

